\title{
Heart Failure by Invasive Diagnostic Technique
}

National Cancer Institute

\section{Source}

National Cancer Institute. Heart Failure by Invasive Diagnostic Technique. NCI

Thesaurus. Code C119209.

A finding of heart failure through an invasive diagnostic technique. 